\title{
DYNAMICS AND COMPOSITION OF RINGS
}

\author{
BRUNO SICARDY \\ Observatoire de Paris and Université Pierre et Marie Curie, Meudon, F-92195, France
}

Received: 16 April 2004; Accepted in final form: 12 October 2004

\begin{abstract}
Planetary rings are found around all four giant planets of our solar system. These collisional and highly flattened disks exhibit a whole wealth of physical processes involving dust grains up to meter-sized boulders. These processes, together with ring composition, can help understand better the formation and evolution of proto-satellite and proto-planetary disks in the early solar system. The present chapter reviews some fundamental aspects of ring dynamics and composition. The forthcoming exploration of the Saturn system by the Cassini mission will bring both high resolution and time-dependent information on Saturn's rings.
\end{abstract}

Keywords: Rings, disks, dynamics, planetary formation

\section{Planetary Rings}

Planetary rings consist in extremely thin disks of innumerable colliding particles revolving around a central planet. They are found around all the four giant planets of our solar system, Jupiter, Saturn, Uranus and Neptune. They exhibit a wide variety of sizes, masses and physical processes. For instance, spiral waves spanning several tens of km, and akin to galactic features, are observed in Saturn's dense rings. At the other end, dust grains submitted to electromagnetic or radiation forces are observed to evolve over a few days only in some tenuous regions of planetary rings.

A complete review of planetary rings clearly remains out of the scope of this chapter. Instead, we would like to address here a few basic issues related to planetary rings, and see how the forthcoming Cassini mission to Saturn may help solve some of these issues.

On the long term, one would like to understand better the connections between the rings global parameters (mass, optical depth, etc...) and their local properties (particle size and distribution, velocity dispersion, etc...). Such connections can eventually give clues on the accretion and fragmentation mechanisms which lead to the formation of satellites or planets in the early solar system, or in other protoplanetary disks. 


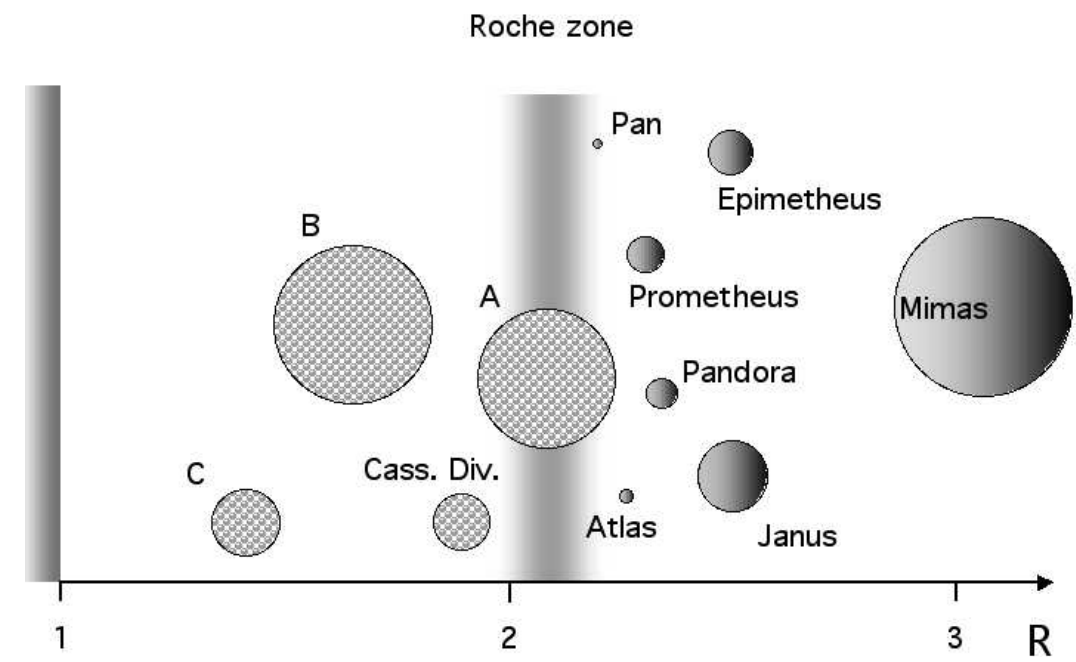

Figure 1. The relative masses of Saturn's inner satellites and rings as a function of their distance to the planet center, in units of the planet radius. The "sizes" of the rings have been calculated by lumping all the material of A, B, C rings and Cassini Division into single bodies (see Esposito, 1993). All the sizes have been plotted so that to respect the relative masses of the various bodies involved. For comparison, Mimas has a diameter of about $500 \mathrm{~km}$. Note the decrease of satellite sizes as one approaches the Roche limit.

\section{Rings around Giant Planets}

All giant planets are surrounded by rings. Detailed reviews of the dynamical and physical properties of these systems can be found in Borderies et al. (1984), Harris (1984), Ward (1984), Nicholson and Dones (1991), Goldreich (1992), Esposito (1993) and Cuzzi (1995). More specific reviews are available for each ring system, see for instance Cuzzi et al. (1984) and Esposito et al. (1984) for Saturn's rings, Burns et al. (2004) for Jupiter's rings, Smith et al. (1986), Esposito et al. (1991) and French et al. (1991) for Uranus' rings, and Smith et al. (1989) and Porco et al. (1995) for Neptune's rings. Finally, general reviews on rings oriented toward the Cassini mission can be found in Cuzzi et al. (2002) and Esposito (2002).

All these rings differ in mass by various orders of magnitude. Only Saturn's rings have an integrated mass comparable to those of significant satellites like Mimas or Encelade. For instance, lumping all Saturn's rings into a single body would yield a satellite with a diameter of the order of $500 \mathrm{~km}$, see Figure 1.

We see in this figure that smaller and smaller satellites are encountered as one gets closer to the planet, a natural consequence of tidal stress. Also, these small satellites tend to be underdense, as they have densities in the range $0.4-0.6 \mathrm{~g} \mathrm{~cm}^{-3}$ (Rosen et al., 1991; Nicholson et al., 1992). This indicates that they are probably loose aggregates of icy material, possibly accumulated in the outer regions of the rings, and then driven outward by tidal forces raised by the latters. 


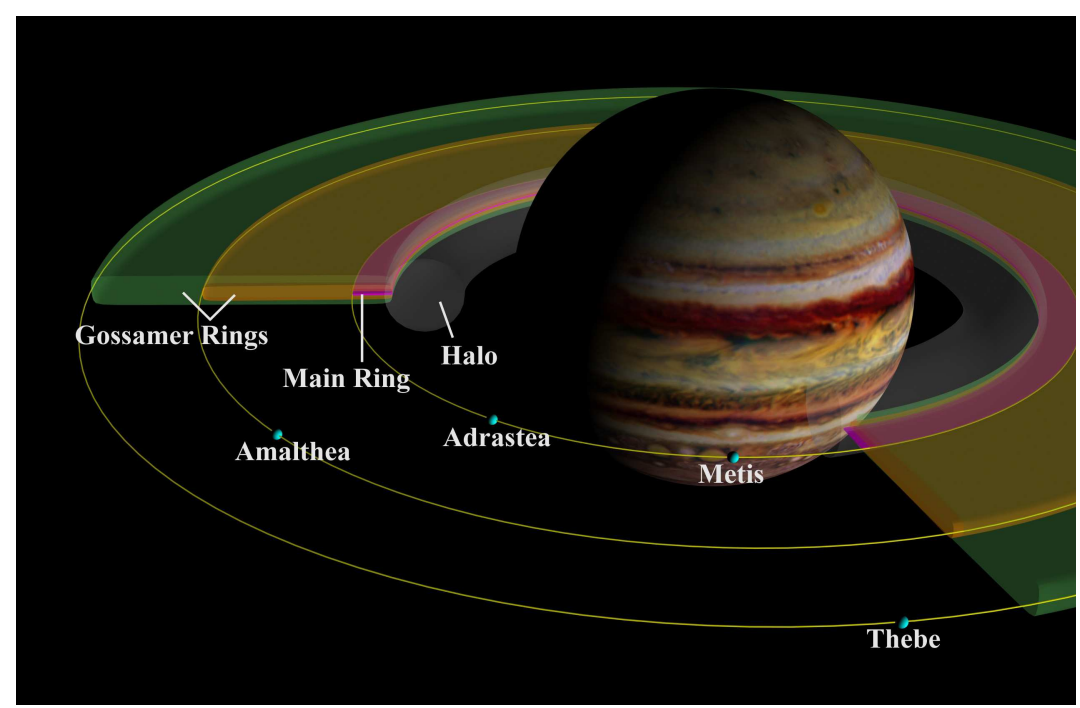

Figure 2. This cartoon depicts schematically the structure of dusty Jupiter rings (taken from http://photojournal.jpl.nasa.gov). The inner main ring (pink) is associated with the small moons Adrastea and Metis, while the outer gossamer rings (yellow and green) are respectively produced by impacts on Amalthea and Thebe (see Burns et al., 2004). The innermost ring (grey) is a very tenuous $3 \mathrm{D}$ torus produced by dust interacting with Jupiter's magnetic field.

As one gets in the Roche zone of the planet, satellites and rings co-exist, and finally, only collisional rings are found, as tidal forces overcome self-gravity of the satellites.

The other planetary rings exhibit the same general behavior as in Figure 1, i.e. smaller and smaller satellites near the Roche limit, then a mixture of rings and satellites, then only rings (Nicholson and Dones, 1991). However, the cumulated masses of these rings are much smaller than those of Saturn.

For instance, gathering Uranus' observable rings together would amount to a satellite of no more than some tens of $\mathrm{km}$ in size, while the same operation would yield a km-sized boby for Neptune's rings, and a lump of some tens of meters at most in the case of Jupiter* (see Esposito, 1993).

Some particularities are associated with each ring system, among which we can quote:

- Jupiter's rings. They are extremely tenuous, as stated above. The dust that we see in these rings is short lived and appears to be associated with small Jovian moons, from which it is excavated, see the discussion below and in Burns et al. (2004), as well as Figure 2.

- Uranus' rings. Although some of Uranus' rings are tenuous and wide, several of them are on the contrary very dense (with optical depth up to $\tau>1$ ), but

* These mass estimates do not take into account possible small satellites or large chunks embedded in those rings, and not discovered yet. 
very narrow (1 to $100 \mathrm{~km}$ depending on the rings), with extremely sharp edges and solid precession, thus requiring very efficient confinement mechanisms.

- Neptune's rings. Besides some dusty tenuous rings, Neptune possesses ring arc features which span only 40 degrees in longitude, out of the 360 degrees available. Although such a configuration is unstable in a matter of months, it has been maintained for more than 20 years now. Again, this requires very specific confining mechanisms, as explained later.

\section{Ring Dynamics}

We can divide the physical processes at work in the rings into two main areas.

One area concerns the large (cm-sized or larger) particles which suffer frequent collisions, leading to a continuous chain of accretion/fragmentation phenomena, plus collective behaviors associated with gravitation and collisions. These processes cause among others a viscous spreading of the rings, as well as a secular exchange of angular momentum with nearby satellites. The time scales associated with these processes are relatively long, typically a million years for instance for the viscous spreading of narrow Uranus' rings, to some hundred of millions years for the collapse of Saturn's A ring onto the B ring through the tidal torque from Mimas (Goldreich and Tremaine, 1982; Borderies et al., 1984). These scales could be compared to geological times scales on the Earth. Note, however, that these time scales remain short compared to the age of the solar system.

Another area concerns microscopic particles (mm-sized down to a few micrometers), for which electromagnetic and radiation forces are much more important. The lifetime of these particles is very short (a few hours to a few millenia at most), due to re-accretion, plus radiation and electromagnetic forces (Burns et al., 2004). Consequently, permanent sources must be invoked to explain their presence. Taking again an Earth analog, these processes could be compared to those encountered in meteorology or oceanography, for which time scales of some weeks or months are frequent.

Of course, the two domains described above are not disconnected, as dust can permanently be re-accreted on large particles, while the latters can be disrupted at any moment into small grains due to a collision.

\subsection{LARge PARTICLES}

\subsubsection{Roche Limit}

Loosely speaking, rings are found inside the Roche zone of the central planet, see Figure 1 . This can be easily understood as tidal stresses become more important near the planet. Reality is more complex, though, as cohesive forces can allow a satellite to live inside the Roche zone, while collisions can grind a satellite into a ring outside that zone. 
The resulting limit between rings and satellites is then a compromise between self-gravity, tensile strength, surface forces, velocity dispersion, particle sizes, etc. . . In particular it happens that rings and satellites can co-exist in the same region, a feature exhibited in all ring systems.

Even in those regions when only rings are found, the local behavior of the particles can be rather complex, and the very notion of a single particle can become pretty fuzzy. In some cases, there could even be the possibility of liquid and solid phases co-existing in the rings, with narrow regions where colliding particles have large velocity dispersions, and narrow regions where they are stuck together in a rigid manner (Tremaine, 2003).

\subsection{Disk Stability}

A detailed discussion and review on disks stability can be found in Binney and Tremaine (1988), from which we extract here some relevant results connected to planetary rings.

Generally, a circumplanetary disk tends to collapse under the influence of collisions, which dissipates energy while conserving angular momentum: the disk is actually the configuration of least energy for a given angular momentum. The collapse, however, does not continue for ever down to an infinitely thin disk of surface density $\Sigma_{0}$, as instabilities then show up. The finite size and masses of the larger particles actually maintain a finite velocity dispersion $c_{s}$ (i.e. a finite "temperature") in the disk.

This temperature maintains a pressure in the disk, which prevents the gravitational collapse of the smallest scales. A dimensional analysis using the quantities $c_{s}$ and $\Sigma_{0}$ shows that we can obtain a length by writing $c_{s}^{2} / G \Sigma_{0}$. More precisely, it can be shown disturbances with spatial scales $\lambda$ smaller than:

$$
\lambda_{J}=\frac{c_{s}^{2}}{G \Sigma_{0}}
$$

will be stabilized (i.e. will not gravitationally collapse). It can be shown that $\lambda_{J}$ is the 2-D version of a Jeans wavelength.

On the other hand, in a disk rotating at angular velocity $\Omega$, large structures of scale $\lambda$ display differential velocities of order $\lambda \Omega$. These structures will then spin more and more rapidly as they collapse, reaching a rotational barrier at some point. A new length can be obtained by writing $G \Sigma_{0} / \Omega^{2}$. More precisely, disturbances larger than:

$$
\lambda_{R}=\pi^{2} \frac{G \Sigma_{0}}{\Omega^{2}}
$$

are stabilized against gravitational collapse by the disk rotation, which can be seen as a 2-D version of the Roche limit.

The disk is thus stable for disturbances with scales smaller than $\lambda_{J}$ and larger than $\lambda_{R}$. The quantity $Q=\sqrt{\lambda_{J} / \lambda_{R}}$ is called the Toomre parameter (Toomre, 
1964). Consequently, the disk is stable at all scales if $\lambda_{J}>\lambda_{R}$, i.e. if:

$$
Q=\frac{c_{s} \Omega}{\pi G \Sigma_{0}}>1 .
$$

The disk actually adjusts itself so that to be just at the limit $Q \sim 1$, i.e. to be in a state of marginal instability. If for instance $Q$ is smaller than unity (cold disk), then gravitational instabilities build up, the sizes of the largest particles increase, which gravitationally stirs the whole system and eventually increases $c_{s}$ so that $Q \sim 1$, at which point instabilities disappear. If on the contrary $Q$ is too large (hot disk), then collisions cause fragmentation and dissipation finally damps $c_{s}$ so that to reach again $Q \sim 1$.

For $Q=1$, the size of the marginally unstable disturbance is:

$$
\lambda_{T}=2 \pi^{2} \frac{G \Sigma_{0}}{\Omega^{2}}=2 \frac{c_{s}^{2}}{G \Sigma_{0}}=2 \pi \frac{c_{s}}{\Omega}=2 \pi h,
$$

where $h=c_{s} / \Omega$ is the thickness of the disk. There are various ways to express $h$. A physical approach is to note that $m_{r}=\pi a^{2} \Sigma_{0}$ the total mass available in the ring, where $a$ is the typical radius of that ring. The expressions above then yield:

$$
h \sim a \times \frac{m_{r}}{M},
$$

where $M$ is the mass of the central planet.

Typical values of Saturn's rings surface density (in the densest regions) yield $m_{r} / M \sim 10^{-7}$, while $a \sim 10^{5} \mathrm{~km}$, so that $h$ is of the order of a some tens of meters. This result agrees with independent and indirect measurements of wave propagation in Saturn's rings, and appear as a natural result of marginal stability in a self-gravitating collisional disk.

Furthermore, the marginally instability scale $\lambda_{T}$ is of the order some hundreds of meters, and is probably the explanation for the quadrant asymmetries observed in Saturn's A ring (Colombo et al., 1977).

Finally, the thickness of some tens of meters quoted above must be maintained by the gravitational stirring of the largest particles of the rings, of the order of the escape velocity at the surface of those particles, $v_{\text {lib }}=\sqrt{2 G m_{l} / R_{l}}$, where $m_{l}$ and $R_{l}$ are respectively the mass and radius of the largest particles. Equating $v_{\mathrm{lib}}$ to $c_{s}=h \Omega$ yields:

$$
R_{l} \sim \sqrt{\frac{3 \Omega^{2}}{8 \pi \rho_{l}}} h,
$$

where $\rho_{l}$ is the density of the largest particles. Loose agregates of icy particles have typical densities of $\rho_{l} \sim 100-1000 \mathrm{~kg} \mathrm{~m}^{-3}$, yielding $R_{l} \sim$ of a few meters, consistent again with Voyager radio experiments (Marouf et al., 1983). 


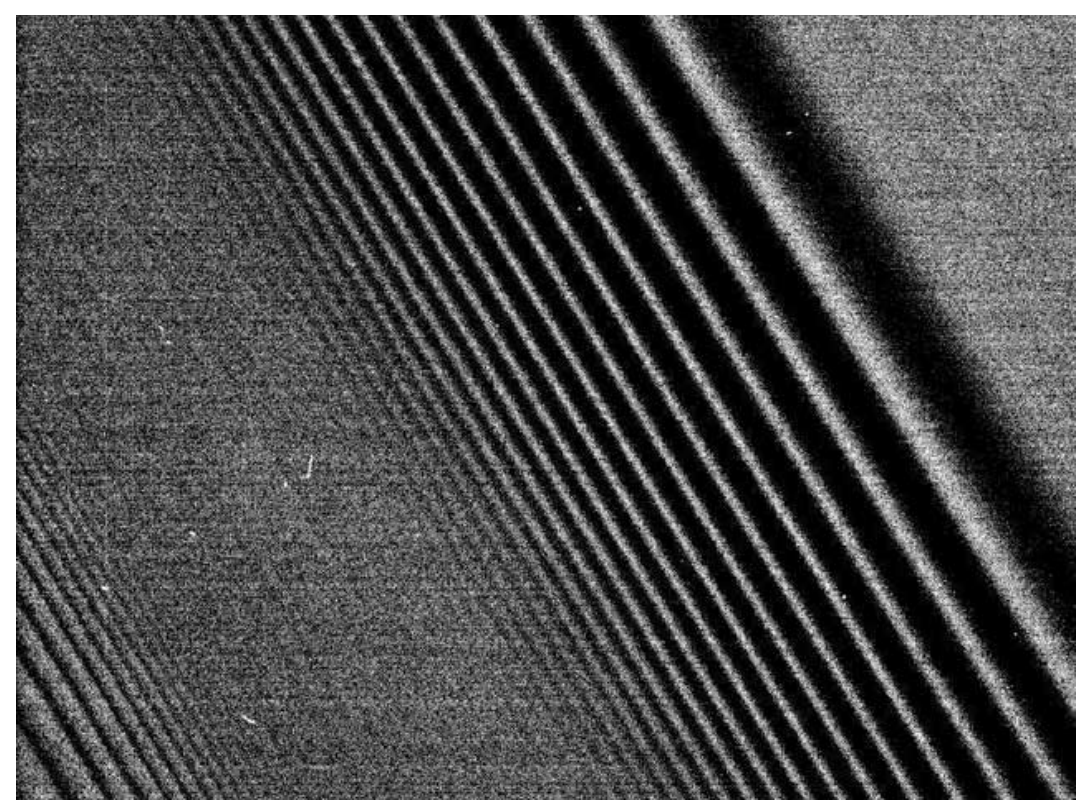

Figure 3. An image of Saturn's dark (unlit) A ring taken by the narrow angle camera on the Cassini spacecraft during its orbit insertion (1st of July 2004, picture taken from http://antwrp.gsfc.nasa.gov/apod/ap040705.html). A bending wave excited by the Mimas 5:3 resonance is visible in the upper right, while a density wave associated with the same resonance appears in the lower left corner. Both wave trains yield important clues on the local physical properties of the rings, see text.

\subsubsection{Resonances}

Many instances of resonances are encountered in planetary rings. They usually involve commensurabilities between the orbital frequencies of ring particle and those of some satellites. Other resonances (the so-called Lorentz resonances) involve commensurabilities between the rotation of the planet magnetic field and the orbital motion of charged particles, see especially the case of Jupiter (Hamilton and Burns, 1993; Burns et al., 2004).

\subsection{SPIRAL WAVES}

Resonances amplify the perturbing effect of satellite on the rings. As such, they drive macroscopic effects in the rings. In particular, collective interactions between the particles (due to self gravity of the disk and to collisions) result in spiral wave modes excitation of several tens of $\mathrm{km}$ in the radial direction, an easily resolved spatial scale for spacecraft like Voyager or Cassini.

These features are unique and precious probes of the microscopic properties of the rings, like the kinematic viscosity $v$ or the local surface density $\Sigma$, see the review by Shu (1984) and Figure 3. 
For instance, the spacing between consecutive crests in density waves can provide the surface density of the disk, which amounts to $\Sigma \sim 70 \mathrm{~g} \mathrm{~cm}^{-3}$ in Saturn's A ring (Esposito et al., 1983; Esposito et al., 1984). Similarly, the damping distance of bending waves yields the kinematic viscosity $v$, which is in turn connected to the velocity dispersion, and eventually, to the vertical thickness of the rings, yielding $h \sim 10-50 \mathrm{~m}$ (Ibid.).

Another important consequence of resonances is that they allow the rings to secularly exchange angular momentum and energy with the exciting satellites, see Goldreich and Tremaine (1982). Although complicated in the details, the value of the resonant torque is independent (at least in the linear case) of viscosity, pressure and surface densities over a wide range of values, see Meyer-Vernet and Sicardy (1987). This allows one to give robust estimations of the angular momentum flow between the satellites and the rings.

This flow is much more efficient that tides raised by satellites on the planet. For instance, Saturn's A ring is expected to be pushed towards B ring over typical time scales of some $10^{8}$ years due to resonances with Mimas, a time short compared to the age of the solar system (Goldreich and Tremaine, 1982).

\subsection{SHARP EDGES}

Another noteworthy effect of resonances between rings and satellites is to truncate in some cases the rings, ensuring the maintenance of very sharp edges of no more that $100 \mathrm{~m}$ in the radial direction, while these features should be rapidly destroyed due to the viscous spreading of the particles. Examples of such edges are encountered in the outer part of Saturn's A and B rings, as well as in Uranus' rings.

This sharpness is due to the local inversion of the viscous angular momentum flow for those streamlines in the ring which lie very nearby (a few $\mathrm{km}$ ) the resonance with the moon (Borderies et al., 1989). This is an interesting example of a very efficient confining mechanism, which may have applications for protoplanetary disk perturbed by already formed planets. Another interesting consequence of highly disturbed streamlines is to potentially ensure the rigid precession of eccentric narrow rings like those of Uranus, see Chiang and Goldreich (2000).

However, most of the sharp features in rings, especially those of Saturn, are not associated with resonances. This means that some physics is still missing to explain the abundance of fine scale structures in rings. An interesting possibility in that some of these features in dense rings are caused by phase transitions between solid-like and fluid-like particle organization, see Tremaine (2003).

\subsection{RADiAL CONFINEMENT}

The exchange of angular momentum between the rings and the satellites at resonances is such that a satellite always tends to push the ring away from it own orbital location. Thus, two satellites are in principle able to confine a ring between them 
against viscous spreading, the so-called shepherding mechanism (Borderies et al., 1984).

This is indeed observed in several circumstances, see e.g. the Uranian $\epsilon$ ring, whose both edges are confined by the small satellites Ophelia and Cordelia.

Many of the observed narrow rings, however, are not observed to be shepherded by two satellites, either because the latters are too small to be discovered at present, or because some physics is not yet understood. For instance, a single satellite could in some cases confine a narrow ring (Goldreich et al., 1995). In other cases, like Saturn's F ring, two satellites (Prometheus and Pandora) are observed on each side of the ring, but they can hardly confine it in a conventional way as their distances to the ring do not match the expected values predicted by the shepherding mechanism.

\subsection{Azimuthal Confinement}

Several examples of arc-like feature are observed in planetary rings, but they are generally short lived. For instance, the features observed in Saturn's F rings during the 1995 ring plane crossing do not survive more than a month or so (Showalter, 2004), and are probably the results of collisions between the members of a so far invisible belt of larger parent bodies (Cuzzi et al., 1984; Poulet et al., 2000).

An exception to that rule is the Neptune's ring arc system. Since their discovery in 1984 (Hubbard et al., 1986), the arcs have been observed again in the following years, either from the ground (Sicardy et al., 1991; Nicholson et al., 1995) or from spacecraft (Smith et al., 1989). Even after ten years, they maintain their basic structure (Dumas et al., 1999; Sicardy et al., 1999), although some changes are detectable. Such time scales are much longer than the time it would take for the Keplerian shear to destroy the arcs, an affair of a few month in the absence of an active azimuthal confinement mechanism.

Among the most promising explanation for this kind of confinement is the action of the so-called corotation resonance sites, akin to the well known Lagrange points $L_{4}$ or $L_{5}$ of a satellite, but not necessarily sharing the orbit of that satellite (Goldreich et al., 1986). Such a resonance has been identify in the case of Neptune's arcs (Porco, 1991), but more subtle effects, including the mass of the arcs itself, must be taken into account for a satisfying model for these features to be fully consistent (Namouni and Porco, 2002).

\subsection{SMALl PARTicles}

Small particles in rings are submitted to non-gravitational forces which drastically change their dynamics when compared to large particles. While in the latter case collisions and self gravity play an essential role, in the former case the important processes are, among others:

- Electromagnetic forces on charged particles, leading to important effects of the planet magnetic field on the dust particle population (see e.g. Grün et 
al., 1984), including the so-called Lorentz resonances (Hamilton and Burns, 1993).

- Radiation forces and Poynting-Robertson drag due to the solar photons (and to a lesser extent, the planet radiation), see the detailed review by Burns et al. (1979).

- Sticking on and ejection from a so-called regolith layer deposited on larger particles (Poulet et al., 2000).

The time scales associated with these processes are in general much shorter than those exhibited by large particles. Actually, time scales as short as a few days are encountered in the evolution of dust, e.g. in Saturn's F ring (Showalter, 2004). Thus, in order for dust to be observed within planetary rings, permanent sources must be invoked.

These sources are thought to be small satellites, or large particles, which are too faint to be detected by imagery, but large enough to provide the released dust over large time scales. Actually, a stationary state could be reached in some cases, where the dust released during inter-particle collisions or meteoroid bombardment, could be re-accreted by the large particles, thus forming a regolith layer on the latters (Cuzzi and Burns, 1988; Poulet et al., 2000).

In others cases, dust rings are clearly associated with known satellites, see for instance the case of Saturn's E ring with Enceladus (Hamilton and Burns, 1994). An extreme case is offered by Jupiter's rings as observed from the ground and by the Galileo spacecraft. While Thebe and Amalthea closely shepherd the main ring, Adrastea or Metis appear to be the main source of particles for the Gossamer ring (de Pater et al., 1998; Burns et al., 2004). In those cases, the dusty rings reach a stationary state where the dust removed by electromagnetic and radiation forces (or satellite sweeping), is replaced by dust produced through meteoroid bombardment of the parent moons, or by collisions between the particles. In such a configuration, the source satellites must be large enough to produce enough dust through bombardment, but not too large for allowing the dust to escape after an impact. This compromise could explain why all satellites are not able to maintain a tenuous dusty ring.

\section{Composition}

Due to their brightness, Saturn's rings are so far the only ones for which relatively high resolution spectra are available. For other rings, only broad band photometry is presently possible, making the determination of the composition problematical.

Concerning Saturn's rings, the presence of water ice have been detected from several decades (Pilcher et al., 1970). Actually, it is difficult to detect something else than water ice in these rings. Recent works (Poulet and Cuzzi, 2002; Poulet et al., 2003) underline the importance of surface texture and small amount of contaminants for interpreting correctly Saturn's rings spectra, especially in the UV. 


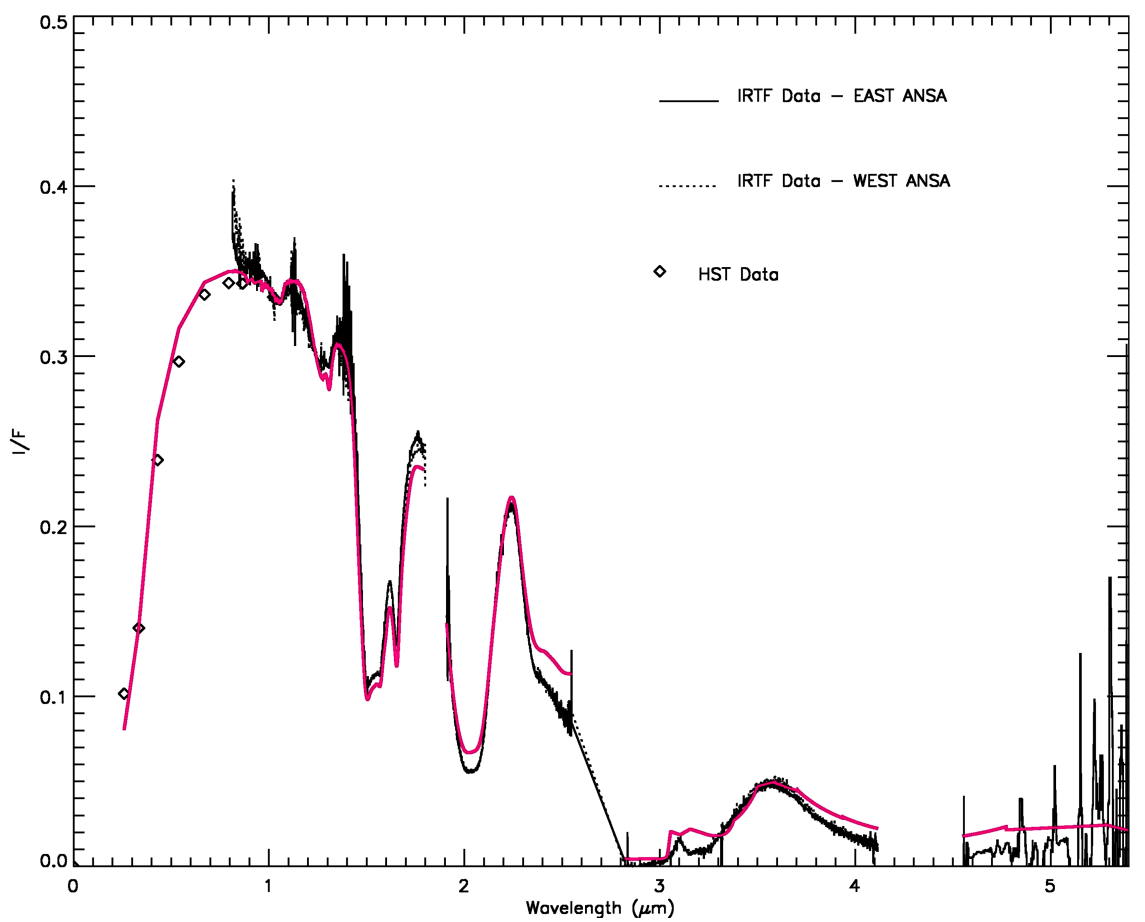

Figure 4. Spectrum of Saturn's B ring taken at $4.7^{\circ}$ phase angle, compared with its best-fit model (red curve). The ring particle surface is made of dirty water ice grains of different sizes (typically 10 , 100, 1000 micrometers) contaminated by red organic material (taken from Poulet et al., 2003).

An overall fit to Saturn's B ring spectrum by these latter authors (see Figure 4) shows for instance that the observations are well reproduced if the particles are covered with an intimate (i.e. 'salt-and-pepper') mixture of grains with different typical sizes, with a discrete size distribution near three different values, namely 10,100 , and $1000 \mu \mathrm{m}$. Among them, $95 \%$ or so are grains with a few percents of refractory organic solid (tholin) impurities, and 5\% are coarse grains of a dark material composed of amorphous carbon.

\section{Cassini Observations}

The present chapter illustrates the variety and complexity of ring dynamics. While the Voyager missions brought a huge crop of results during the few weeks of encounters, they nevertheless missed time for a more profound look to rings to be taken. The Cassini tour will have several advantages with respect to Voyager: first better instruments as technology improved between the 1970's and the 1990's, and second a four year nominal mission, i.e. much more time available for science.

Among some highlighting actions that Cassini can take, and without being exhaustive, we can quote: 
- Better constrain the particles size distribution using photometry at all wavelengths (from UV to radio) and all phase angles. Other methods (aureole imaging, stellar and solar occultations, etc...) can also be very useful for this purpose.

- Get the global composition and its "geographical" variations using spectra at all wavelengths (from UV to IR). This may be a challenge, as water ice largely dominates the ring spectrum.

- Follow in real time the evolution of short-lived features like the F ring clumps, the spokes, arc features, or the chaotic behavior of Prometheus and Pandora, etc... This could turn out to be a very important step for understanding better the long term evolution of rings.

- Get the sizes and densities of the small satellites, in particular the underdense small satellites near the rings. By the same token, discover new populations of small satellites near the rings, as the present limit ( $5 \mathrm{~km}$ or so) is fixed by the Voyager cameras capabilities, and not by ring dynamics itself.

\section{References}

Binney, J. and Tremaine, S.: 1988, Galactic Dynamics, Princeton University Press.

Borderies, N., Goldreich, P., and Tremaine, S.: 1984, 'Unsolved problems in planetary ring dynamics', in R. Greenberg and A. Brahic (eds.), Planetary rings, The University of Arizona Press, pp. 713-734.

Borderies, N., Goldreich, P., and Tremaine, S.: 1989, 'The formation of sharp edges in planetary rings by nearby satellites', Icarus 80, 344-360.

Burns, J.A., Lamy, P.L., and Soter, S.L.: 1979, 'Radiation forces on small particles in the solar system', Icarus 40, 1-48.

Burns, J.A., Hamilton, D.P., Nicholson, P.D., de Pater, I., Ockert-Bell, M.E., and Thomas, P.C.: 2001, 'The formation of Jupiter's faint ring', Science 284, 1446-1450.

Burns, J.A., Simonelli, D.P., Showalter, M.R., Hamilton, D.P., Porco, C.C., Esposito, L.W., and Throop, H.: 2004, 'Jupiter's Ring-Moon System', in F. Bagenal, T. Dowling, and W. McKinnon (eds.), Jupiter: Planet, Satellites and Magnetosphere, Cambridge University Press, in press.

Chiang, E.I, and Goldreich, P: 2000, 'Apse alignment of narrow eccentric planetary rings', Astron. J. 540, 1084-1090.

Colombo, G., Goldreich, P., and Harris, A.W: 1977, 'Spiral structure as an explanation for the asymmetric brightness of Saturn's A ring', Nature 264, 344-345.

Cuzzi, J.N., Lissauer, J.J., Esposito, L.W., Holberg, J.B., Marouf, E.A., Tyler, G.L., and Boischot, A.: 1984, 'Saturn's rings: properties and processes', R. Greenberg and A. Brahic (eds.), Planetary rings, The University Of Arizona Press, pp. 73-199.

Cuzzi, J.N. and Burns, J.A.: 1988, 'Charged particle depletion surrounding Saturn's F ring: Evidence for a moonlet belt', Icarus 74, 284-324.

Cuzzi, J.N.: 1995, 'Evolution of planetary ringmon systems', Earth, Moon, and Planets 67, 179-208.

Cuzzi, J.N., et al.: 2002, 'Saturn's rings: pre-Cassini status and mission goals', Space Sci. Rev. 104, 209-251.

de Pater, I., Showalter, M.R., Burns, J.A., Nicholson, P.D., Liu, M.C., Hamilton, D.P., Graham, J.R.: 1996, 'Keck infrared observations of Jupiter's ring system near Earth's 1997 ring plane crossing', Icarus 138, 214-223. 
Dumas, C., Terrile, R.J., Smith, B.A., Schneider, G., and Becklin, E.E: 1999, 'Stability of Neptune's ring arcs in question', Nature 400, 733-735.

Esposito, L.W., O'Callaghan, M., and West, R.A.: 1983, 'The structure of Saturn's rings: implications from the Voyager stellar occultation', Icarus 56, 439-452.

Esposito, L.W., Cuzzi, J.N., Holberg, J.B., Marouf, E.A., Tyler, G.L., and Porco, C.C.: 1984, 'Saturn's rings: structure, dynamics and particle properties', T. Gehrels and M.S. Matthews (eds.), Saturn, The University of Arizona Press, Tucson, pp. 463-545.

Esposito, L.W., Brahic, A., Burns, J.A., and Marouf, E.A.: 1991, 'Particles properties and processes in Uranus' rings', in J.T. Bergstralh, E.D. Miner, and M.S. Matthews (eds.), Uranus, The University of Arizona Press, Tucson, pp. 410-465.

Esposito, L.W.: 1993, 'Understanding planetary rings', Annu. Rev. Earth Planet. Sci. 21, 487-523.

Esposito, L.W.: 2002, 'Planetary Rings', Rep. Prog. Phys. 65, 1741-1783.

French, R.G., Nicholson, P.D., Porco, C.C., and Marouf, E.A.: 1991, 'Dynamics and structure of the Uranian rings', in J.T. Bergstralh, E.D. Miner, and M.S. Matthews (eds.), Uranus, The University of Arizona Press, Tucson, pp. 327-409.

Goldreich, P.: 1992, 'Puzzles and prospects in planetary ring dynamics', S. Ferraz-Mello (ed.), Chaos, resonance and collective dynamical phenomena in the solar system, Kluwer Academic Publishers, Dordrecht Boston London, pp. 65-73.

Goldreich, P., and S. Tremaine, S.: 1982, 'The dynamics of planetary rings', Ann. Rev. Astron. Astrophys 20, 249-283.

Goldreich, P., Tremaine, S., and Borderies, N.: 1986, 'Towards a theory for Neptune's arc rings', Astron. J. 92, 490-494.

Goldreich, P., Rappaport, N., and Sicardy, B.: 1995 'Single-sided shepherding', Icarus 118, 414-417.

Grün, E., Morfill, G.E., and Mendis, D.A.: 1984, 'Dust-magnetosphere interactions', in R. Greenberg and A. Brahic (eds.), Planetary rings, The University of Arizona Press, Tucson, pp. 275-332.

Hamilton, D.P. and Burns, J.A.: 1993, 'Lorentz and gravitational resonances on circumplanetary particles', Adv. Space Res. 13, 241-248.

Hamilton, D.P. and Burns, J.A.: 1994, 'The origin of Saturn's E ring: self-sustained, naturally', Science 264, 550-550.

Harris, A.W.: 1984, 'The origin and evolution of planetary rings', in R. Greenberg and A. Brahic (eds.), Planetary rings, The University of Arizona Press, pp. 641-659.

Hubbard, W.B., Brahic, A., Sicardy, B., Elicer, L.R., Roques, F., and F. Vilas: 1986, 'Occultation detection of a Neptunian ring-like arc', Nature 319, 636-640.

Marouf, E.A., Tyler, G.L., Zebker, H.A., Simpson, R.A., and Eshleman, V.R.: 1987, 'Particle size distributions in Saturn's rings from Voyager 1 radio occultation', Icarus 54, 189-211.

Meyer-Vernet, N. and Sicardy, B.: 1987, 'On the physics of resonant disk-satellite interaction', Icarus 69, 157-175.

Namouni, F. and Porco, C.: 2002, 'The confinement of Neptune's ring arcs by the moon Galatea', Nature 417, 45-47.

Nicholson, P.D. and Dones, L.: 1991, 'Planetary rings', Rev. Geophys. 29, 313-327.

Nicholson, P.D., Hamilton, D.P., Matthews, K., and Yoder, C.F.: 1992, 'New observations of Saturn's coorbital satellites', Icarus 100, 464-484.

Nicholson, P.D., Mosqueira, I., and Matthews, K.: 1995, 'Stellar occultation observations of Neptune's rings', Icarus 113, 293-330.

Pilcher, C. B., Chapman, C.R., Lebofsky, L.A., and Kieffer, H.H.: 1970, 'Saturn's rings: Identification of water ice', Science 167, 1372-1373.

Porco, C.: 1991, 'An explanation for Neptune's ring arcs', Science 400, 995-1001.

Porco, C.C., Cuzzi, J.N., Esposito, L.W., Lissauer, J.J., and Nicholson, P.D.: 1995, 'Neptune's rings', in D.P. Cruikshank (ed.), Neptune and Triton, The University of Arizona Press, Tucson, pp. 703804. 
Poulet, F., Sicardy, B., Nicholson, P.D., Karkoschka, K., and Caldwell, J.: 2000, 'Saturn's ring-plane crossings of August and November 1995: a model for the new F-ring objects', Icarus, 144, 135148.

Poulet, F. and Cuzzi, J.N.: 2002, 'The Composition of Saturn's Rings', Icarus 160, 350-358.

Poulet, F., Cruikshank, D.P., Cuzzi, J.N., Roush, T.L., and French, R.G.: 2003, 'Compositions of Saturn's rings A, B, and C from high resolution near-infrared spectroscopic observations', Astron. Astrophys. 412, 305-316.

Rosen, P.A., Tyler, G.L., Marouf, E.A., and Jack J. Lissauer, J.J.: 1991, 'Resonance structures in Saturn's rings probed by radio occultation: II. Results and interpretation', Icarus 93, 25-44.

Showalter, M.R.: 2004, 'Disentangling Saturn's F Ring I. Clump Orbits and Lifetimes', Icarus, submitted.

Shu, F.H.: 1984, 'Waves in planetary rings', R. Greenberg and A. Brahic (eds.), Planetary rings, The University of Arizona Press, pp. 513-561.

Sicardy, B., Roques, F., and A. Brahic: 1991, 'Neptune's rings, 1983-1989: ground-based stellar occultations observations. I. Ring-like detections', Icarus 89, 220-243.

Sicardy, B., Roddier, F., Roddier, C., Perozzi, E., Graves, J.E., Guyon, O., and Northcott, M.J.: 1999, 'Images of Neptune's ring arcs obtained by a ground-based telescope', Nature 400, 731-733.

Smith, B.A., et al.: 1986, 'Voyager 2 at Uranus in the Uranian system: Imaging science results', Science 233, 43-64.

Smith, B.A., et al.: 1989, 'Voyager 2 at Neptune: Imaging Science results', Science 246, 1422-1449. Toomre, A.: 1964, 'On the gravitational stability of a disk of stars' Astrophys. J. 139, 1217-1238.

Tremaine, S.: 2003, 'On the Origin of Irregular Structure in Saturn's Rings', Astron. J. 125, 894-901.

Ward, W.: 1984, 'The solar nebula and the planetesimal disk', in R. Greenberg and A. Brahic (eds.), Planetary rings, The University of Arizona Press, Tucson, pp. 660-684.

Address for Offprints: Bruno Sicardy, Observatoire de Paris, LESIA, bâtiment 10, 5, Place Jules Janssen, 92195 Meudon Cédex, France; bruno.sicardy@obspm.fr 